\title{
IMAGERIE \\ photoacoustique biomédicale
}

\author{
Sergey VILOV ${ }^{1}$, Thomas CHAIGNE ${ }^{2,3}$, Bastien ARNAL $^{1}$, Emmanuel BOSSY $^{1}$ \\ ${ }^{1}$ Univ. Grenoble Alpes, CNRS, LIPhy, Grenoble, France \\ ${ }^{2}$ Charité Universitätsmedizin Berlin and Humboldt University, Einstein Center for Neuroscience, \\ NeuroCure Cluster of Excellence, Berlin, Germany \\ ${ }^{3} \mathrm{CNRS}$, Institut Fresnel, Marseille, France \\ emmanuel.bossy@univ-grenoble-alpes.fr
}

Depuis le début des années 2000, l'imagerie photoacoustique connait un formidable essor en tant que technique d'imagerie optique biomédicale. Cet essor, qui se traduit à la fois par les nombreuses recherches menées par un nombre d'équipes de plus en plus important dans le monde et par les multiples applications qui en découlent, est lié aux performances uniques de cette modalité d'imagerie optique en termes de résolution et de contraste.

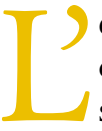
effet photoacoustique, qui consiste en l'émission de son suite à l'absorption de lumière au travers d'effets thermoélastiques, a été découvert à la fin du $19^{\mathrm{e}}$ siècle par Alexander Graham Bell, l'inventeur du téléphone. Ce n'est cependant qu'avec l'avènement du laser dans les années 60 qu'ont pu se développer les premières applications de l'effet photoacoustique, dans le domaine de la spectroscopie. Les premières publications suggérant l'utilisation de cet effet pour l'imagerie des milieux biologiques sont apparues au début des années 80 dans le domaine radiofréquence, suivies quelques années plus tard des premières preuves de concepts dans le domaine visible [1]. Dans cet article, nous présentons dans un premier temps le principe général de l'imagerie photoacoustique dans les tissus biologiques, en insistant sur la nature du contraste des images photoacoustiques et sur les différentes modalités en termes de résolution. Nous présentons ensuite quelques dispositifs expérimentaux parmi les plus répandus, dont nous illustrons les performances à partir d'images issues de publications récentes. Avant de conclure, nous présentons brièvement quelques résultats récents obtenus dans le contexte de l'imagerie super-résolue.

\section{Principe général de l'imagerie photoacoustique}

De façon générale, l'image photoacoustique est reconstruite à partir de la mesure de signaux acoustiques engendrés par absorption de la lumière envoyée sur l'échantillon. Pour des raisons liées à l'efficacité de la génération photoacoustique, on utilise généralement pour l'imagerie biomédicale des impulsions brèves nanosecondes pour illuminer le tissu à imager, et on se limite ici à cette situation : l'absorption de la lumière impulsionnelle crée une élévation rapide de température dans le tissu mou qui engendre par effet thermoélastique une élévation de pression, pression qui relaxe alors en entrainant la propagation d'ondes acoustiques impulsionnelles dans le tissu. Pour des impulsions nanosecondes, et pour des absorbeurs de dimensions supérieures à quelques microns (ce qui est le cas pour tous les vaisseaux sanguins comme pour un globule rouge individuel), les ondes acoustiques ne se propagent qu'une fois l'impulsion de lumière absorbée : on parle de régime de confinement de contrainte, régime qui pour des impulsions nanosecondes optimise l'amplitude de l'onde acoustique émise tout en restant dans le cadre des limites d'illumination admissibles sur la peau (de l'ordre de la dizaine $\mathrm{de} \mathrm{mJ} / \mathrm{cm}^{2}$ par impulsion nanoseconde). Dans ce cadre, les élévations de température sont tout
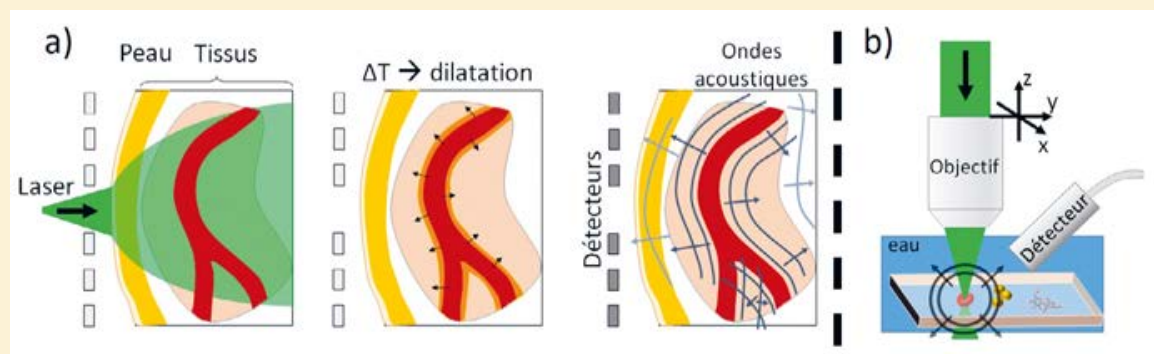

Figure 1. (a) Imagerie photoacoustique à résolution acoustique. La lumière diffuse en profondeur dans le tissu ; la résolution est conditionnée par le contenu fréquentiel des signaux acoustiques détectés et la diffraction acoustique. (b) Imagerie photoacoustique à résolution optique : l'image est obtenue à partir du balayage point par point d'un faisceau focalisé en surface de l'échantillon, et le signal acoustique détecté mesure l'absorption optique au point de focalisation, dont les dimensions sont limitées par la diffraction optique. 


\section{AVEC NOTRE EXPERTISE, VOUS ALLEZ VOIR LA POLARISATION DANS TOUS SES ÉTATS !}

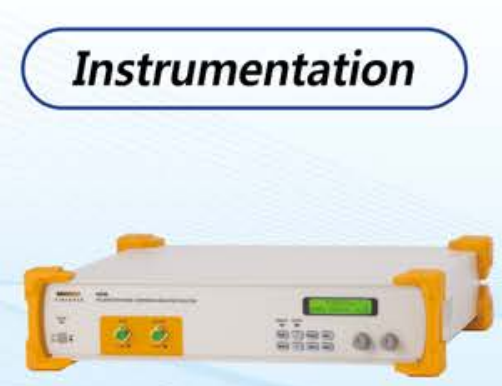

PMD Emulator

Simuler l'effet de la PMD

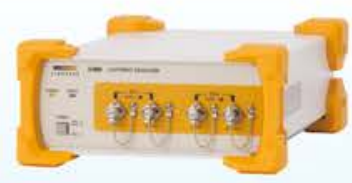

ASE Source

Large bande avec une excellente planéité

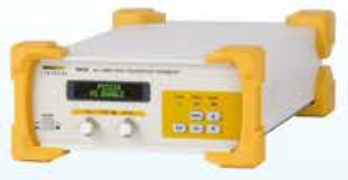

Polarization Scramber Supprimer l'effet de la polarisation

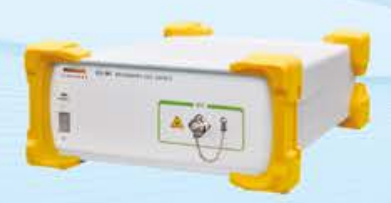

Lightwave Equalizer

Créer des filtres de formes arbitraires

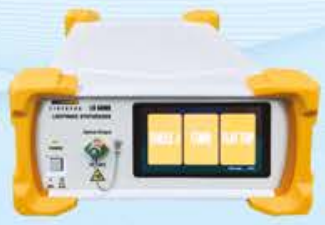

Lightwave Synthesizer

Source incohérente multifonctionnelle : mono longueur d'onde, peigne, très aplatie

\section{Composants}

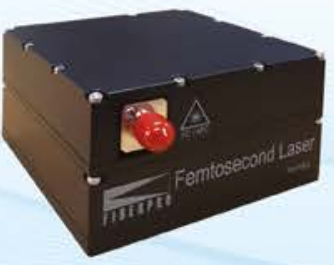

Laser à fibre pulsé à blocage de mode passif Facile à utiliser
Femtosecond Mode -Locked Fiber Laser

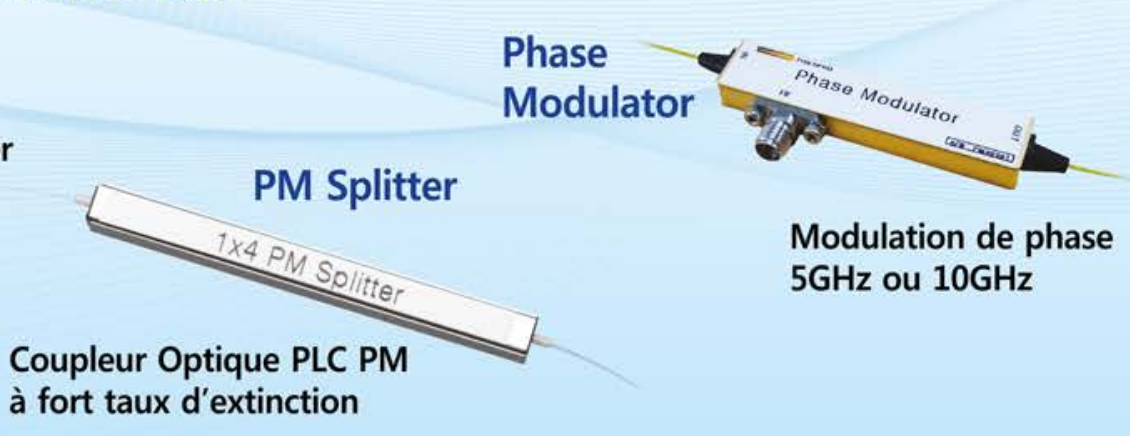

Contrôle ou brouillage de porarisation à grande vitesse

\section{LN PC}

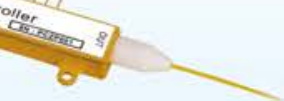

Pour plus d'information, merci de contacter notre partenaire WAVETEL

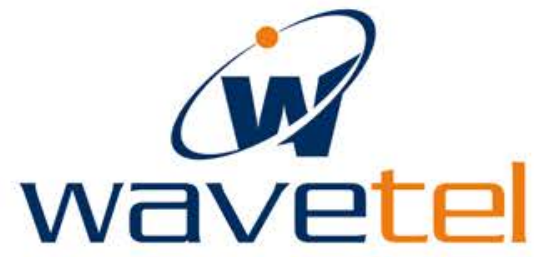

a simac group company

\section{www.wavetelfit}

PARIS - RENNES - LORIENT 
au plus de l'ordre de quelques millièmes de degrés. Si les signaux mesurés sont de nature acoustique, l'imagerie photoacoustique fournit bien des images des propriétés optiques d'absorption, et fait donc partie de la famille des techniques d'imagerie optique. Une de ses caractéristiques fondamentales est sa sensibilité spécifique à l'absorption optique. En particulier, comme nous le détaillons par la suite, elle est le plus souvent mise en cuvre pour l'imagerie de la vascularisation sanguine, et à ce titre ne nécessite pas d'agents de contraste exogènes, un atout considérable par rapport à des techniques telles que l'imagerie de fluorescence. Mais l'essor de l'imagerie photoacoustique a été motivé principalement par les performances de la technique en termes de résolution : en effet, la formation résolue d'une image suppose de pouvoir localiser la région de l'échantillon qui a émis un son. On distingue dans ce cadre deux approches fondamentalement distinctes quant à la façon dont est construite l'image photoacoustique, et les performances en termes de résolution et de profondeur d'imagerie sont en conséquence très différentes.

\section{Imagerie photoacoustique à résolution acoustique}

Lorsqu'on illumine un tissu biologique avec de la lumière visible, la pénétration de cette dernière en profondeur dans le tissu est limitée principalement par le phénomène de diffusion. En effet, le libre parcours moyen de transport dans les tissus biologiques est de l'ordre du millimètre pour la lumière visible: si un photon peut se propager sur plusieurs centimètres avant d'être absorbé, sa trajectoire dans un tissu biologique s'apparente à une marche aléatoire au-delà d'une profondeur de l'ordre du millimètre. C'est ce phénomène de diffusion qui limite les techniques de microscopie purement optiques à des profondeurs d'imagerie de quelques centaines de microns, dans la mesure où elles reposent sur une hypothèse de propagation balistique des photons (propagation en ligne droite). L'effet photoacoustique a été proposé pour pallier la limite de

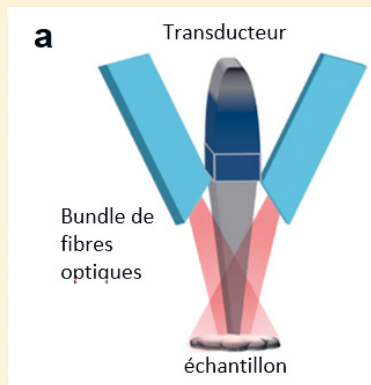

b

C

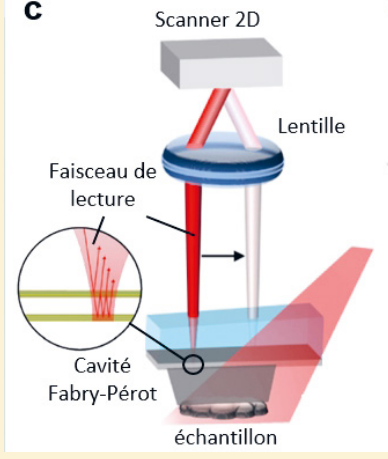

d

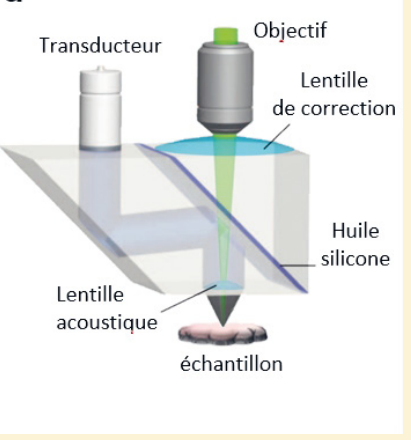

Figure 2. Quelques exemples de configurations pour l'imagerie

photoacoustique. (a)-(c) Imagerie photoacoustique à résolution acoustique: l'échantillon est illuminé plein champ, et la résolution est celle de limage acoustique reconstruite en mesurant les signaux sur un réseau de capteurs ultrasonores, de façon tout à fait analogue à l'imagerie échographique. (d) Imagerie photoacoustique à résolution optique : un transducteur unique, focalisé pour augmenter sa sensibilité, mesure les signaux en provenance du point de focalisation du faisceau excitateur. Figure adaptée de [2] et [3]. résolution liée à la diffusion optique : en effet, contrairement à la lumière, le son n'est pratiquement pas diffusé dans les tissus biologiques mous, qui s'apparentent donc à des milieux quasiment transparents aux ondes acoustiques. De plus, la vitesse du son dans les tissus mous s'avérant quasiment homogène (de l'ordre de grandeur de la vitesse du son dans l'eau, $1500 \mathrm{~m} \mathrm{~s}^{-1}$ ), on peut alors effectuer l'image de la distribution des sources de son à l'intérieur d'un tissu mou, comme on le ferait en imagerie optique avec des photons balistiques. À la différence de l'imagerie optique où on utilise des lentilles pour reformer une image de la distribution de sources dans le plan d'imagerie, l'imagerie (ultra)sonore repose sur la détection résolue en temps des ondes acoustiques. À l'aide d'un réseau de capteurs ultrasonores et connaissant la vitesse du son, on peut alors par des méthodes équivalentes à de la triangulation connaitre la position d'une source de son à partir des signaux acoustiques reçus. C'est ce principe qui est utilisé de façon très similaire en imagerie échographique et imagerie photoacoustique à résolution acoustique. En imagerie photoacoustique on détecte les sons émis par les absorbeurs optiques, quand en imagerie échographique on détecte les sons rétrodiffusés par les tissus. Les images ainsi formées étant basées sur la détection d'ondes acoustiques, la résolution des images obtenues n'est limitée que par la diffraction des ondes acoustiques: on parle ainsi d'imagerie photoacoustique à résolution acoustique.

Le principe de l'imagerie photoacoustique à résolution acoustique est illustrée sur la figure 1a. Une impulsion illumine la surface du tissu et diffuse en son sein : en profondeur, au-delà typiquement d'un millimètre, la lumière est étalée par le phénomène de multidiffusion, mais se propage en un temps qui reste de l'ordre de quelques nanosecondes pour des profondeurs de l'ordre de quelques centimètres. Les zones qui absorbent la lumière (les vaisseaux sanguins par exemple, comme illustré sur la figure) vont alors par effet thermoélastique être mises sous contrainte et émettre des ondes sonores. Le temps d'illumination restant très bref à l'échelle de la propagation ultrasonore, tout se passe comme si les absorbeurs étaient illuminés de façon instantanée. On peut alors montrer que les ondes acoustiques émises sont des impulsions dont le contenu fréquentiel est inversement proportionnel à la taille des absorbeurs, avec la vitesse du son comme coefficient de proportionnalité: un vaisseau sanguin d'une centaine de micron (resp. d'un millimètre) de 
autour de la dizaine de $\mathrm{MHz}$ (resp. autour d'1 MHz). Les signaux reçus par les capteurs ultrasonores sont alors analysés pour reconstruire la distribution des sources de son, reliée à la distribution d'absorption optique. La résolution de l'image photoacoustique va dépendre du contenu fréquentiel qui peut être détecté par les capteurs ultrasonores : celui-ci est limité par l'absorption des ultrasons dans les tissus qui croit avec la fréquence et la profondeur. Comme pour l'imagerie échographique, la gamme de fréquences détectables et donc la résolution de l'imagerie photoacoustique dépendent de la profondeur d'imagerie : en pratique, on constate que le rapport entre la profondeur d'imagerie et la résolution acoustique correspondante est de l'ordre de 200 (à $2 \mathrm{~cm}$ de profondeur, la résolution de l'imagerie photoacoustique est ainsi de l'ordre de $100 \mu \mathrm{m}$ ).

\section{Microscopie photoacoustique à résolution optique}

Pour des profondeurs superficielles pour lesquelles il reste possible de focaliser la lumière, on peut réaliser une microscopie photoacoustique à résolution optique, tout à fait analogue aux microscopies optiques à balayage: comme illustrée sur la figure $1 b$, chaque impulsion est focalisée point par point sur la surface de l'échantillon, et on mesure le son émis en provenance de la zone focale optique par un capteur ultrasonore unique. Dans ce cas, la résolution est donnée par le confinement du faisceau d'excitation, limité par la diffraction optique, de l'ordre de $1 \mu \mathrm{m}$ dans le plan $x y$ de balayage. Cette approche est strictement identique dans son principe à une microscopie de fluorescence à balayage, et n'en differe que par la nature de la mesure et du contraste obtenu:l'intérêt principal de la microscopie photoacoustique à résolution optique est de fournir une image spécifique de l'absorption optique.

\section{Dispositifs expérimentaux}

La figure 2 illustre les configurations expérimentales les plus répandues en imagerie photoacoustique. Les configurations (a), (b) et (c) fournissent des images photoacoustiques à résolution acoustique (illumination plein champ) et ne différent que par le type de capteurs ultrasonores et par leur disposition dans l'espace. Sur la figure $2 a$, la lumière est envoyée sur l'échantillon par un faisceau de fibres optiques solidaires d'un réseau linéaire de capteurs ultrasonores. La barrette ultrasonore détecte alors les signaux photoacoustiques émis dans son plan d'imagerie, et l'image reconstruite est une image bidimensionnelle. L'avantage de cette approche est qu'elle peut être mise en œuvre sur un échographe ultrasonore et une sonde d'échographie standard auxquels il suffit d'adjoindre une source lumineuse. Elle est cependant limitée à des images bidimensionnelles, qui de plus sont souvent sujettes à de nombreux artefacts : il se peut par exemple que le plan d'imagerie contienne des sources de sons émis principalement hors du plan d'imagerie, rendant alors ces sources invisibles dans l'image photoacoustique. Pour pallier ces effets liés
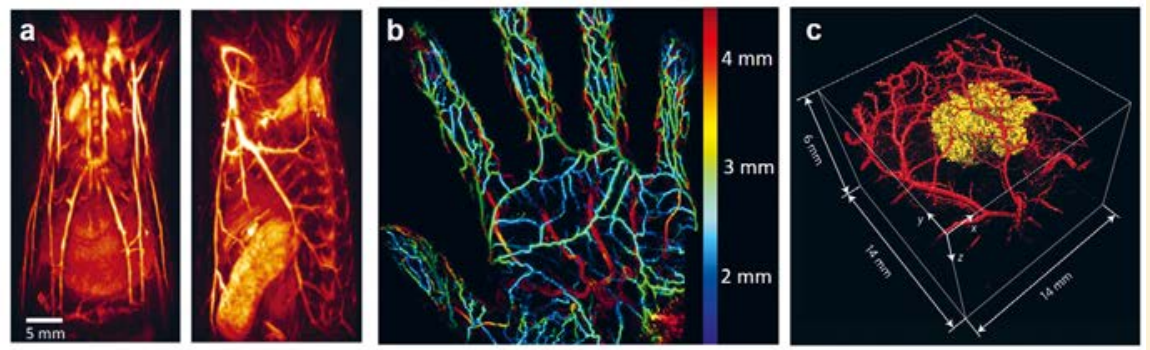

Figure 3. Quelques exemples d'image photoacoustique in vivo à résolution acoustique. (a) Images de projection de la distribution d'absorption d'une souris [3]. (b) Image de projection codée en profondeur de la vascularisation d'une main [4]. (c) Image d'une tumeur entourée d'un réseau vasculaire [5].
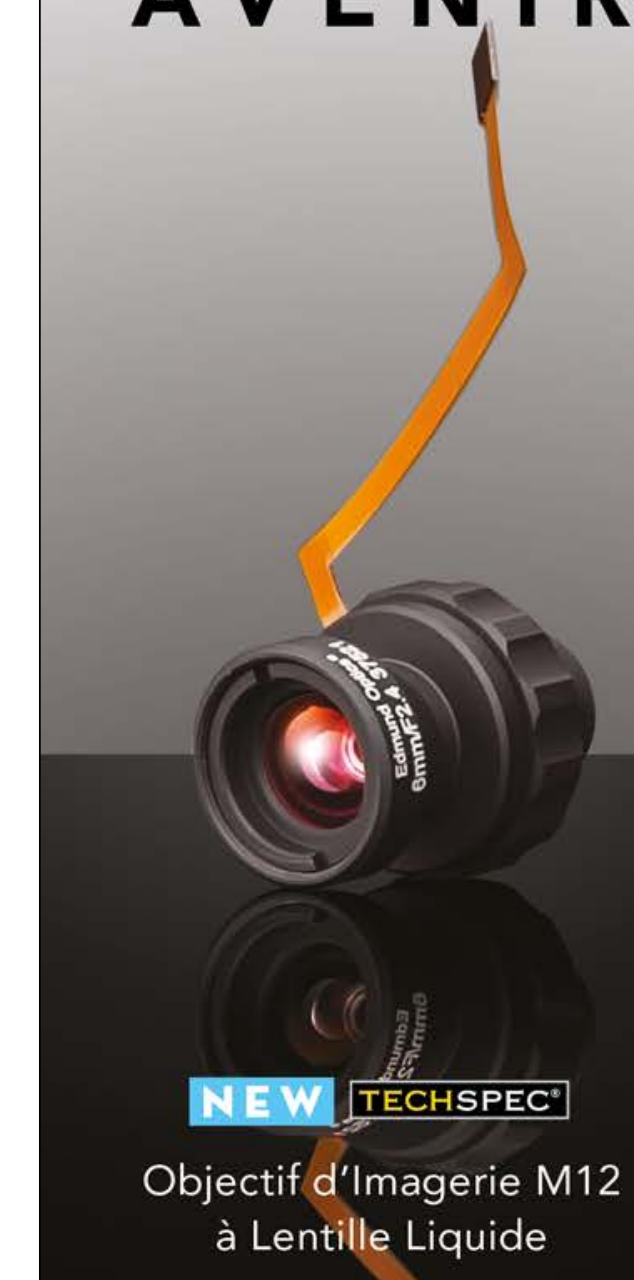

Objectif $d^{\prime}$ Imagerie $M 12$ à Lentille Liquide TECHSPEC $^{\circ}$ - une mise au point rapide à différentes distances de travail. Les nouveaux objectifs M12 sont spécifiquement conçus pour les lentilles liquides Varioptic et sont disponibles avec quatre distances focales de 6 à $16 \mathrm{~mm}$. Les conceptions $f / 2,4$ haute résolution recouvrent les capteurs grand format jusqu'à $1 / 1,8^{\prime \prime}$.

Pour en savoir plus :

www.edmundoptics.fr/M12 
à une détection incomplète du champ ultrasonore, des approches tomographiques ont été développées pour lesquelles l'intégralité du champ acoustique rayonné est acquis séquentiellement en déplaçant mécaniquement le réseau de capteurs ultrasonores tout autour de l'échantillon à imager, comme illustré sur la figure $2 b$. Une fois acquis l'ensemble des signaux émis, on peut alors reconstruire une image tridimensionnelle de l'échantillon. Une majorité des équipes de recherche utilise pour mettre en œuvre ces deux approches des réseaux de capteurs piézo-électriques semblables voire identiques à ceux utilisés pour l'imagerie échographique conventionnelle. Comme en imagerie ultrasonore, la fréquence centrale des capteurs est adaptée à la profondeur d'imagerie souhaitée, typiquement $\mathrm{du} \mathrm{MHz}$ à quelques dizaines de $\mathrm{MHz}$ pour des profondeurs de quelques centimètres à quelques millimètres. La figure $2 c$ illustre une approche « tout-optique », pour laquelle la détection des ondes ultrasonores se fait également optiquement : le capteur est ici constitué d'un interféromètre Fabry-Pérot dont l'épaisseur varie lors du passage d'une onde ultrasonore, variations qui se traduisent par une modulation d'intensité réfléchie d'un faisceau optique focalisé en un point de l'interféromètre. En balayant point par point le faisceau focalisé sur la surface de l'interféromètre, on synthétise ainsi un capteur multiélément permettant alors une reconstruction tridimensionnelle de l'échantillon. L'intérêt principal de cette approche est lié au caractère largebande de la mesure optique, qui permet de reconstruire les images photoacoustiques avec moins d'artefacts que les approches basées sur des capteurs piézo-électriques dont le caractère résonant favorise artificiellement dans les images certaines tailles d'absorbeurs. La complexité de sa mise en œuvre, en comparaison des méthodes de détection piézo-électriques bénéficiant des technologies de l'imagerie ultrasonore, en limite pour l'instant l'utilisation à quelques groupes de recherches.

La figure $2 d$ illustre une manière de mettre en cuvre la microscopie photoacoustique à résolution optique: de façon générale, il convient pour cette approche de détecter avec un maximum de sensibilité le signal acoustique émis par les absorbeurs présents dans la zone de focalisation de la lumière, dont les dimensions sont de l'ordre de quelques microns, conduisant à des signaux relativement faibles en comparaison des approches à résolution acoustique. Pour ce faire, on utilise un capteur ultrasonore focalisé avec une grande surface de détection afin d'augmenter la sensibilité de la détection. Dans l'exemple de la figure 2d, le capteur ultrasonore et l'objectif de microscope sont assemblés de manière confocale de façon à ce que la zone illuminée se trouve toujours au foyer du transducteur ultrasonore. Le prisme de couplage utilisé ici est transparent à la lumière pour laisser passer le faisceau excitateur focalisé, mais réfléchit les ultrasons en direction du détecteur ultrasonore. L'image photoacoustique est alors obtenue en balayant l'ensemble objectif/ transducteur/prisme parallèlement à la surface de l'échantillon, pour fournir une image bidimensionnelle en face de l'échantillon. Comme pour toutes les techniques de microscopie à balayage, un inconvénient majeur de la microscopie photoacoustique à résolution optique concerne le temps d'acquisition qui est proportionnel au champ de vue.

\section{Quelques exemples d'images photoacoustiques}

La figure 3 illustre sur quelques exemples le type d'images quel'on peut obtenir en imagerie photoacoustique à résolution acoustique. La figure $3 a$ présente deux images de projection obtenues à partir d'une reconstruction tridimensionnelle, représentant la distribution d'absorption optique dans une souris. Les signaux ontété acquis in vivo par approche tomographique semblable à l'approche illustrée sur la figure $2 b$, avec un temps d'acquisition de l'ordre de 5 minutes. Cette image illustre bien comment l'absorption optique permet d'imager de nombreux éléments anatomiques à l'échelle du petit animal, et en particulier de mettre en valeur la vascularisation grâce à la très forte absorption endogène fournie par le sang. L'important contraste d'absorption lié au sang est également très bien illustré par l'image de la figure $3 b$, obtenue à l'aide d'une matrice bidimensionnelle de capteurs piézo-électriques (fréquence centrale 2 $\mathrm{MHz}$ ). Contrairement à l'approche tomographique qui requiert de multiples acquisitions sous plusieurs angles de vue, une seule acquisition est nécessaire ici, mais seuls les vaisseaux orientés dans le plan de l'image sont visibles. La figure $3 c$ montre l'image d'une tumeur et de son environnement vasculaire, obtenue à l'aide de l'approche tout-optique de la figure $2 c$. La résolution associée à chacune de ces images d'absorption optique (typiquement de la centaine de $\mu \mathrm{m}$ au $\mathrm{mm}$ ) à des profondeurs de l'ordre de quelques $\mathrm{mm}$ à quelques centimètres est une caractéristique unique de l'imagerie par effet photoacoustique.

La figure 4 présente deux exemples d'images obtenues par microscopie photoacoustique à résolution optique, à l'aide du dispositif illustré sur la $f$ gure $2 d$ : sur la figure $4 a$, la résolution de l'ordre du micron est suffisante pour imager la morphologie individuelle des globules rouges, qui apparaissent avec un très fort contraste sur l'image photoacoustique. On retrouve également ce très fort contraste sur l'image de la figure $4 b$, obtenue à l'échelle du réseau vasculaire d'un cerveau de souris imagé in vivo. L'image de la figure $4 b$ fournit de plus une information fonctionnelle, au-delà de
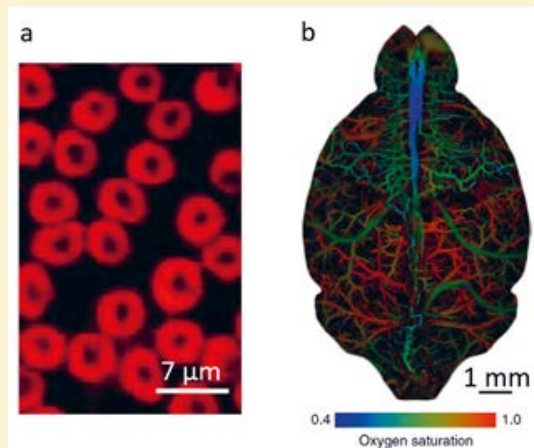

Figure 4. Exemples d'images photoacoustiques à résolution optique. (a) Image de globules rouges uniques. (b) Image in vivo de la saturation en oxygène de l'hémoglobine dans un cerveau de souris. Images tirées de [2]. 


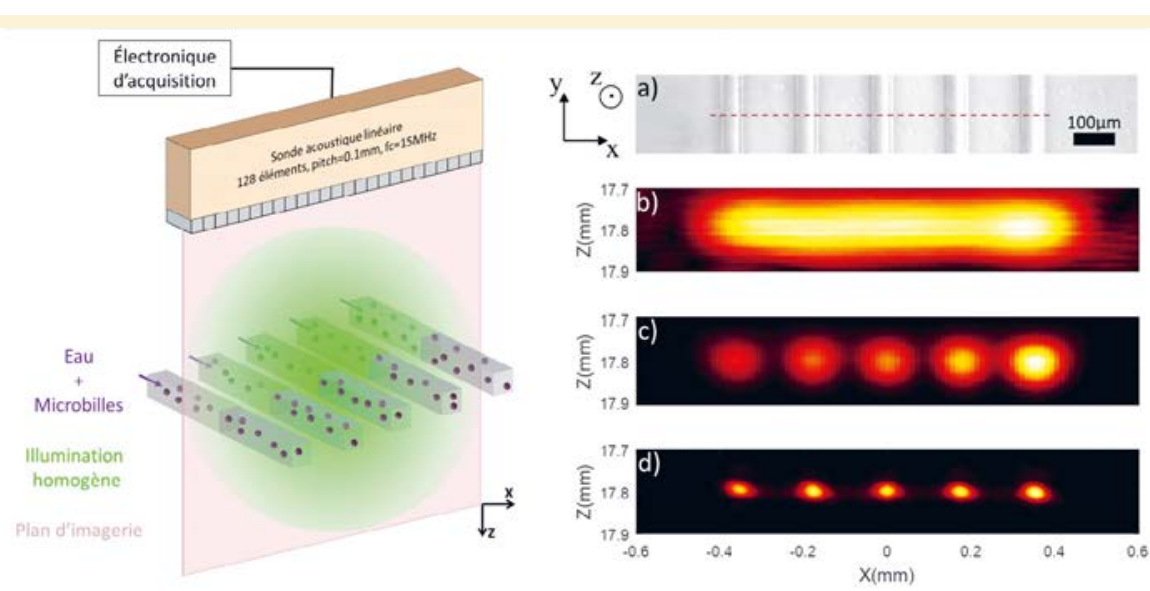

Figure 5. Exemple d'imagerie super-résolue [6,7]. À gauche : schéma du dispositif expérimental. À droite : (a) photographie des canaux microfluidiques; (b) image photoacoustique standard ; (c) image super-résolue obtenue par imagerie de fluctuations (approche SOFI, solution dense d'absorbeurs) ; (d) image super-résolue obtenue par localisation (solution diluée d'absorbeurs).

la simple géométrie du réseau vasculaire: en combinant les images photoacoustiques obtenues à différentes longueurs d'onde, on peut en effet réaliser une spectroscopie d'absorption et ainsi déduire le taux d'oxygénation du sang, traduit sur la figure $4 b$ par l'échelle de couleur.

\section{Vers l'imagerie photoacoustique super-1ésolue}

Un des axes de recherches actuels en imagerie photoacoustique concerne le développement de méthodes permettant d'obtenir des images super-résolues : inspirés par les approches de super-résolution mises en œuvre en optique afin de contourner la limite de diffraction optique, plusieurs groupes travaillent au développement de méthodes d'imagerie photoacoustique en profondeur dont la résolution va au-delà de la limite de diffraction acoustique. La figure 5 présente quelques résultats de principe obtenus in vitro dans notre équipe au Laboratoire Interdisciplinaire de Physique à Grenoble, en imageant des canaux microfluidiques de dimensions typiques des vaisseaux sanguins: la figure $5 b$ illustre une situation où la résolution (photo)acoustique est insuffisante pour distinguer des canaux microfluidiques séparés de $180 \mu \mathrm{m}$ centre à centre. Quand ces canaux microfluidiques sont parcourus par un écoulement de particules absorbantes, l'écoulement conduit à des fluctuations du signal photoacoustique d'une image à l'autre, et on peut alors mettre en œuvre un traitement d'images basé sur ces fluctuations pour obtenir des images super-résolues : l'image de la figure $5 c$ a été obtenue en mesurant les fluctuations statistiques d'ordres supérieurs, en adaptant la méthode initialement proposée en optique pour améliorer la résolution à partir des fluctuations de fluorescence (méthode SOFI). Si les particules absorbantes sont en nombre suffisamment faible, on peut également mettre en œuvre une approche par localisation, consistant à reconstruire l'image photoacoustique en superposant l'ensemble des positions mesurées pour chaque absorbeur

\section{POUR EN SAVOIR PLUS} 8(4), 586-617, 2016 Methods, 13(8), 627, 2016 2198, 2017 Reports, 8(1), 786, 2018 Photonics, 9(4), 239, 2015 isolé, positions dont la mesure n'est affectée que par le niveau de signal et non par la limite de diffraction.

\section{Conclusion}

L'imagerie photoacoustique est une méthode dont les principes de base et la mise en œuvre sont à présent bien établis, comme illustré dans les paragraphes précédents. Le nombre d'équipes de recherche dans le monde travaillant sur ce sujet ne cesse d'augmenter, traduisant un important effort de recherche sur le développement à la fois des méthodes, des instruments et des applications. L'importance de cet effort de recherche commence en particulier à susciter le développement de composants optimisés pour l'imagerie photoacoustique, que ce soit pour l'excitation lumineuse ou la détection acoustique. Si à ce jour, l'essentiel des applications biomédicales in vivo concerne l'imagerie du petit animal, avec plusieurs systèmes commercialisés, plusieurs travaux sont également menés afin de mettre en œuvre l'imagerie photoacoustique sur l'homme, notamment en ajoutant cette modalité sur des imageurs ultrasonores conventionnels. Par la spécificité de ses performances en termes de rapport résolution sur profondeur et de type de contraste, il est à parier que l'imagerie photoacoustique jouera à l'avenir un rôle de plus en plus important au sein de l'éventail des techniques d'imagerie optique.

[1] Manohar, S., Razansky, D. Photoacoustics: a historical review. Advances in Optics and Photonics,

[2] Wang, L.V., Yao, J. A practical guide to photoacoustic tomography in the life sciences. Nature

[3] Deán-Ben, X.L., Gottschalk, S., Mc Larney, B., Shoham, S., Razansky, D. Advanced optoacoustic methods for multiscale imaging of in vivo dynamics. Chemical Society Reviews, 46(8), 2158-

[4] Matsumoto, Y., Asao, Y., Yoshikawa, A., Sekiguchi, H., Takada, M., Furu, M., Toi, M. Label-free photoacoustic imaging of human palmar vessels: a structural morphological analysis. Scientific

[5] Jathoul, A.P., Laufer, J., Ogunlade, O., Treeby, B., Cox, B., Zhang, E., Beard, P.C. Deep in vivo photoacoustic imaging of mammalian tissues using a tyrosinase-based genetic reporter. Nature

[6] Chaigne, T., Arnal, B., Vilov, S., Bossy, E., Katz, O. Super-resolution photoacoustic imaging via flow-induced absorption fluctuations. Optica, 4(11), 1397-1404, 2017

[7] Vilov, S., Arnal, B., Bossy, E. Overcoming the acoustic diffraction limit in photoacoustic imaging by the localization of flowing absorbers. Optics Letters, 42(21), 4379-4382, 2017 